\title{
Design and Implementation of High Precision Data Acquisition System for Offshore Engineering Seismic Exploration Based on Distributed Architecture
}

\author{
Changxian Zhou, Shaopeng Zheng, Youquan Ye, Yonggu Zhang, congrong Ren \\ \{zhouchangxianxm@126.com\}
}

Xiamen Seismic Survey Research Center Xiamen 361021, China

\begin{abstract}
Oil and natural gas are non renewable resources, is an important energy mineral resources and strategic resources, related to the national economic and social development, the relationship between national security. Countries have a fierce competition for oil, the impact of international oil prices on domestic oil prices gradually increased. With the rapid development of China's economy, China's oil and gas gap will be more and more big. In order to alleviate the shortage of oil and gas resources, China has added to the exploration and development of domestic oil and gas. China's sea area is broad, with rich oil and gas resources. Since "eight five", the increment of marine oil has become the main force of oil and gas production in China. The seismic acquisition system of marine high precision towing cable is mainly divided into the underwater acquisition part and the indoor record part. In this paper, we focus on the design and implementation of the terminal part quality control and recording work station software. This paper discusses the specific implementation details of the quality control and recording work station software. The software module includes bus interface design, network communication, data processing, data display, data storage, data drawing, and each QC test algorithm.
\end{abstract}

Keywords: Annular layered structure; SEG-D format; seismic data storage; quality control

\section{Introduction}

Petroleum and natural gas are important energy resources and strategic resources, which are related to the national economic and social development, and the relationship between the national security and the national security. In recent years, the international crude oil prices frequently rise or fall, volatile, from the side of the country to reflect the fierce competition for oil. The influence of international oil price fluctuation on domestic oil price is more and more big. With the rapid development of China's economy, China's oil and gas gap is getting bigger and bigger. According to statistics, in 2020 the basic realization of industrialization, China's oil, natural gas demand gap will be more than $50 \%$. In order to alleviate the shortage of oil and gas resources, China is actively seeking oil from abroad, but also added to the domestic oil and gas exploration and development. China Sea, including the Yellow Sea, Bohai, East China Sea and the South China Sea [1], an area of nearly 3 million square kilometers, is the sacred land of the motherland, is related to the country's sovereignty and rights and interests. At 
present, we are faced with the struggle to safeguard the sovereignty and rights and interests of the state, the core issue is the seabed mineral resources, especially oil and gas. China Sea has 42 dimensions, which is rich in oil and natural gas resources. Only the continental shelf offshore 4130 x $10 \mathrm{sq} \mathrm{km}$ area, there are 10 large-scale Cenozoic Oil and gas bearing basins, such as in the Bohai Sea and the northern Yellow Sea and the South Yellow Sea, East China Sea shelf, West Taiwan, southwest of Taiwan, Pearl River Estuary, Qiongdongnan and Yinggehai Basin and Beibu Gulf. Initial estimate of the amount of resources, oil is 246 tons, natural gas for 12315.7 x $10 \mathrm{~m}$. Since "eight five", the increment of marine oil has become the main force of oil and gas production in China. Although China's sea area is very vast, but due to the complex environment of the sea, to the offshore oil exploration and exploitation has brought great inconvenience. Due to late start, offshore oil exploration technology is not enough, so our country's offshore oil exploration technology is far behind and abroad, also caused our country offshore oil exploration equipment mainly rely on imports of the status quo. On the one hand, the price of imported equipment is more expensive, but not easy to maintain; on the other hand, with the Foreign Company, the use of some of the market in exchange for Foreign Company's technology, the loss is relatively large [2].

\section{Structure of Marine High Precision Towed Cable Seismic Acquisition System}

This chapter mainly of high precision sea streamer seismic acquisition system structure are introduced, in order to give the reader the whole concept of a system, more convenient and understand the position and function of the key quality control and recording software in the whole system. The general situation of the system is introduced from the overall structure and technical index, and the process of the system operation and the task to be completed are understood. The indoor system is the data record and control part of the high precision towed cable seismic acquisition system. This is closely related to the relationship between quality control and recording software, which reflects the functional requirements and tasks of the software.

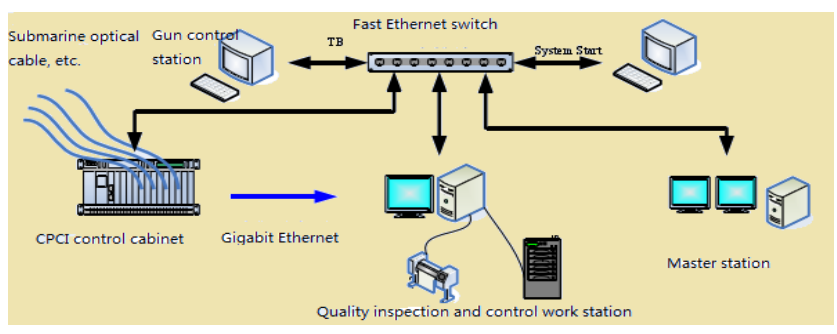

Fig. 1. General structure of the system

\subsection{Master Station}




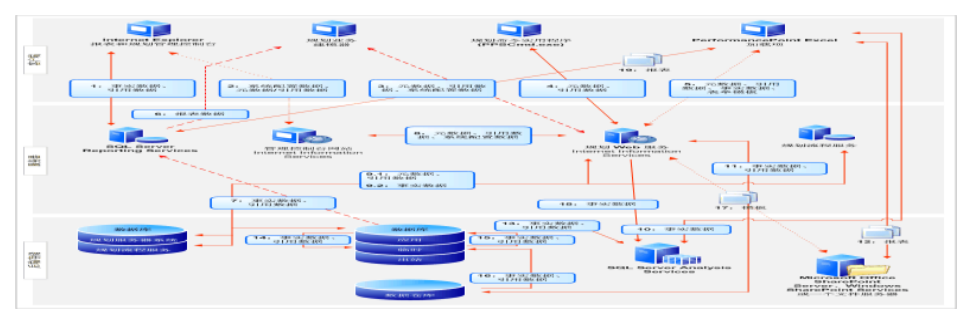

Fig. 2. The master station system structure diagram

The master control station is the core of the system, which is configured and ordered to control the whole seismic exploration system. Master station software is running complete on the overall control of the system of the seismic exploration of the software system, the software provides a good man-machine interface of the system is responsible for the task of the system configuration parameters, task distributing and scheduling, quality system operation monitoring, the system examination and diagnosis and vibrator seismic data graphics real-time display in control workstation. Analyzed from the function, control workstation software can configuration parameters and command control part, a vibrator data record and display part and the auxiliary function (of the system monitoring module and online help module), this part. QR workstation software is responsible for the record control and field quality control of the indoor recording system, which is an important part of the seismic exploration system. Data record control is to achieve the main seismic data to tape targets according to seismic data format real-time write complete collection of marine seismic exploration data, site quality control is for experts in the field staff and exploration to provide a visual interface, to facilitate their real observation of the underwater drag cable acquisition package of waveform data, the analysis of their current working state, and can control the plotter to draw the specified data waveform [3].

\subsection{System Software Design}

QR software runs on the quality control and recording work station, which belongs to the terminal part of the whole system. The performance of the system has a serious impact on the efficiency of the data record. Therefore, the analysis of this software must be reasonable and clear, so that it is conducive to the software debugging, upgrading and maintenance. From the theoretical analysis of building software, more structured, for the preparation of code and code error correction provides a good reference system. This chapter is the overall design of the software, so the software and hardware platform and the overall operation mode of the software are also discussed in detail. Offshore seismic exploration systems mission is geophone acquisition to the seismic signal is converted into an electrical signal, then the signal conversion to digital signal transmission to the indoor system, through the summary and conversion, then the raw data into SEG-D format of seismic data storage to specific memory devices. Quality control and recording station is not only the terminal of the indoor system, but also the terminal of the whole system, the most important responsibility is to collect the data to the storage. So in addition to storing data, the workstation can make more reasonable analysis and explanation of the collected data, which is beneficial to the system acquisition and debugging. Human computer interface is a communication mode of marine seismic exploration personnel and system. So these factors must be taken into account when building software. During the operation, the main control station receives the command sent by 
Ethernet workstation configuration, waiting to receive data. After under the coordination of the control workstation, quality control and recording workstation via Gigabit Ethernet receiving seismic data, receive the navigation information through the Gigabit Ethernet. After recording software to the seismic data format conversion, cache, and then according to the order of the data display, QC test, and data storage [4].

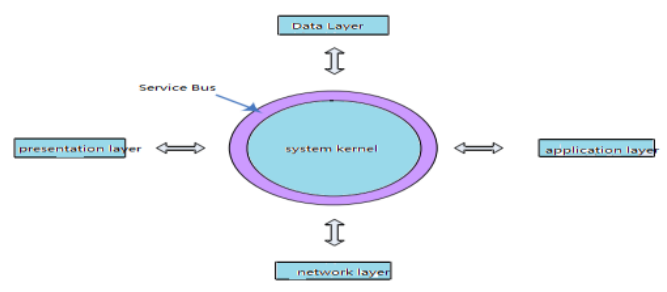

Fig. 3. System software architecture

\subsection{System Data Transmission}

Data layer, presentation layer, application layer and network layer are connected with the kernel through a common peripheral module. When adding peripheral modules, as long as the unified interface can be easily extended to the software. Each of the peripheral modules are independent of each other, do not interfere with each other, the small change caused by the change of code is not spread to the entire code library, so the maintenance of the software is relatively high. For large data transmission, can be increased by increasing the node to share, easy to operate. Therefore, the annular layered architecture has the advantages of strong expansibility, stability, maintainability and operability, and the structure is easy to understand, which is very convenient for the segmentation and integration of the system. The presentation layer is the external view of the software, which is separated from the other layers, and is linked with other levels through the control of the bus and the kernel.

\section{System Network Communication Design}

The interface of the service bus is different from the distributed structure of the single machine and the network. The construction model of the former is the interface of the software, shared memory; the latter will be information transmission on Ethernet of, can be used in C / S mode, in client application by bus interface of information analysis and applications to invoke, because as long as the use of unified interface can be adjusted with, so in different development environment, different programming languages can operation of the distributed system. In this software, each module needs the seismic data information including the number of cables, the number of channels, the sampling points, and the different modules have different control information. The storage module comprises a storage medium selection (magnetic tape or hard disk), and the QC test module comprises a data signal frequency, a signal amplitude, a test item, and a window information. For these different functions for different information, the information received from the net mouth had been included in the and storage in different structure, so the kernel by bus to send the information includes commands and various different information structure pointer. If there is a need, in the bus transfer information can also be added to the synchronization information, to ensure that the 
data of the number of guns in a row. The network communication module only exists and the normal acquisition mode and the QC test mode, this work station mainly carries on the information communication with the main control work station, and carries on the data exchange with the zero slot. In the reading mode, the software does not communicate with the outside world, there is no network communication. We connect the indoor record system to a local area network through the Ethernet, and establish the communication channel as shown below, using the network socket (socket) technology to realize the information transmission between the subsystems. Each pair of communication channels is composed of a number of Client/Server model, in communication with the $\mathrm{QR}$ workstation module, $\mathrm{QR}$ workstations are as the server side [5].

\section{Conclusion}

Engineering is refers to the four cable software into a certain improvement of reliability, stability, availability of the requirements of the project operation. Without reducing the system reliability, stability and other indicators of the premise, the original four cable software for rectification, in order to achieve the best comprehensive performance. This includes the following several aspects: modifications to the original problem: the original four cable system in spite of the sea trials. But there are also more or less of a problem, which is mainly reflected in the stability of the system. Therefore, this part of the rectification of the factors that lead to the instability of the system and solve its problems, so as to enhance the stability of the system. To ensure that the new modified content: the new software system is based on the original delete and modify, for the new join and modify the code part, to ensure that the reliability and stability of this part of the code. Comprehensive index: in order to be able to meet the so-called engineering use, it should be as far as possible according to the geophysical laboratory proposed amendments to the integration of opinion, improve the comprehensive index of the four cable system. With the system debugging, to achieve a higher index.

\section{References}

[1] Meng Qingsheng, Chu Xianfeng, Guo Xiujun, Jia Yonggang, Fan Yuqing. Application of high resolution data processing techniques to seismic exploration in offshore engineering. geophysical progress. No. 03. (2014)

[2] Pei Yanliang, Wang Kuiyang, Li Guanbao, Li Xishuang, Liu Chenguang. Seismic source and application of marine engineering seismic exploration. Petroleum Instrument. No02. (2013)

[3] Hou health, Liu Jiangping. New progress in engineering seismic exploration. exploration of the geophysical progress. No. 06. (2012)

[4] Wu Ming, Chen Guoliang, sun Ninghui. Journal of computer design and implementation of. computer users in parallel environment. No. 10. (2011)

[5] Zhao Hongru, Guo. Progress in engineering survey of. speed rail suppository seismic exploration geophysics. No. 03. (2013) 